\title{
Fiber Optic Methane Sensing System Based on Temperature-tunable Distributed Feedback Laser
}

\author{
Shang-hui Xiao, ${ }^{1 *}$ Yi Jiang, ${ }^{2}$ and Jun Tang ${ }^{3}$ \\ ${ }^{1}$ School of Electronic Engineering, Chengdu Technological University, Chengdu 611730, China \\ ${ }^{2}$ School of Optoelectronics, Beijing Institute of Technology, Beijing 100081, China \\ ${ }^{3}$ Furong Coal Co. Ltd. Attached to Sichuan Coal Group, Yibin 644002, China
}

(Received April 6, 2018; accepted June 11, 2018)

Keywords: fiber optic sensors, methane measurement, absorption spectroscopy, wavelength scanning

A fiber optic methane sensing system based on the temperature-tunable distributed feedback (DFB) laser is experimentally demonstrated. Two laser diodes (LDs) with different wavelengths are used in the system. One LD operates in the wavelength scanning mode, and the other LD is used as the reference beam to eliminate the fluctuation of light intensity. By comparing the intensities of the two wavelengths during wavelength scanning, we can determine methane content. Experimental results show that a measurement resolution of $0.038 \%$ can be reached. The obtained measuring system has good properties of high precision, low cost, and high reliability.

\section{Introduction}

Methane detection has become increasingly important as the coal industry imposes greater demands on the methane measurement and monitoring system. Fiber optic methane sensors have advantages compared with pellistors or catalytic detectors, which detect methane by the ignition of the gas on a heated filament, such as remote monitoring at a far distance, no poisoning, and safety in an explosive and flammable environment. ${ }^{(1,2)}$ Moreover, multiplexing techniques enable many fiber optic sensors to be integrated in one system. Thus, the fiber optic methane monitoring system has attracted considerable interest in recent years. ${ }^{(3-6)}$

The spectrum-absorption-based measurement technique, or absorption spectroscopy, is well suited for monitoring methane gas, owing to its ability to eliminate the perturbation of the light source and the transmission loss caused by dust, water vapor, and so forth. The main content of methane is $\mathrm{CH}_{4}$, and $\mathrm{CH}_{4}$ has two absorption bands at $v_{2}+2 v_{3}$ and $2 v_{3}$, corresponding to the absorption wavelengths of 1.33 and $1.65 \mu \mathrm{m}$, respectively. These two wavelengths locate within low-loss bands of the optical fiber, giving the opportunity to the optical fiber to measure the methane content.

The differential absorption technique has been introduced to increase the performance of the methane sensors. ${ }^{(7-11)}$ In the differential absorption technique, the two wavelengths, one

*Corresponding author: e-mail: xiaosh73@163.com

https://dx.doi.org/10.18494/SAM.2018.1958 
coinciding with one of the absorption lines of methane and the other one shifting slightly away from the absorption line, can be obtained by using two interference filters, ${ }^{(7)}$ a dual-electrode wavelength-tunable distributed feedback (DFB) laser diode (LD), ${ }^{(8)}$ a wavelength-tunable Fabry-Perot filter ${ }^{(8)}$ fiber Bragg gratings $(\mathrm{FBGs}),{ }^{(10)}$ or a fiber loop. ${ }^{(1)}$ In this paper, a fiber optic methane sensing system based on the wavelength-scanning DFB laser that is tuned by temperature is experimentally demonstrated. The measuring system has some good properties of high precision, low cost, and high reliability.

\section{Operation Principle}

The schematic diagram of the measurement setup is shown in Fig. 1. One DFB LD emitting light at the wavelength of $1653 \mathrm{~nm}$ is used as the sensing light, and the operation wavelength locates around an absorption line of $\mathrm{CH}_{4}$. Another DFB LD emits light at the wavelength of $1551 \mathrm{~nm}$, and this beam is used as the reference light. Part of the sensing light is detected directly by PD1 to monitor the intensity fluctuation of the light source, and part of the reference light is detected by PD4 for the same reason. The two beams with different wavelengths are combined by a 1551/1653 nm wavelength division multiplexer (WDM), WDM1. Then, the twobeam light is injected into the gas cell along a long-distance leading fiber. The two beams with different wavelengths go through the same transmission line and gas cell, and thus suffer from the same loss caused by dust, bending loss, vapor, and so forth. The transmitted light is then demultiplexed by another WDM, WDM2, which separates the two beams with different wavelengths by $1551 / 1653 \mathrm{~nm}$. The two beams with different wavelengths are then detected with two photodiodes, PD2 and PD3, respectively. Four output light beams are all detected and sampled into a computer to calculate the methane content. At the sensing wavelength of $1653 \mathrm{~nm}$, the wavelength of the DFB LD scans with a range of $2.5 \mathrm{~nm}$ when the operation temperature increases from 15 to $40{ }^{\circ} \mathrm{C}$. However, the intensity of the sensing LD at the

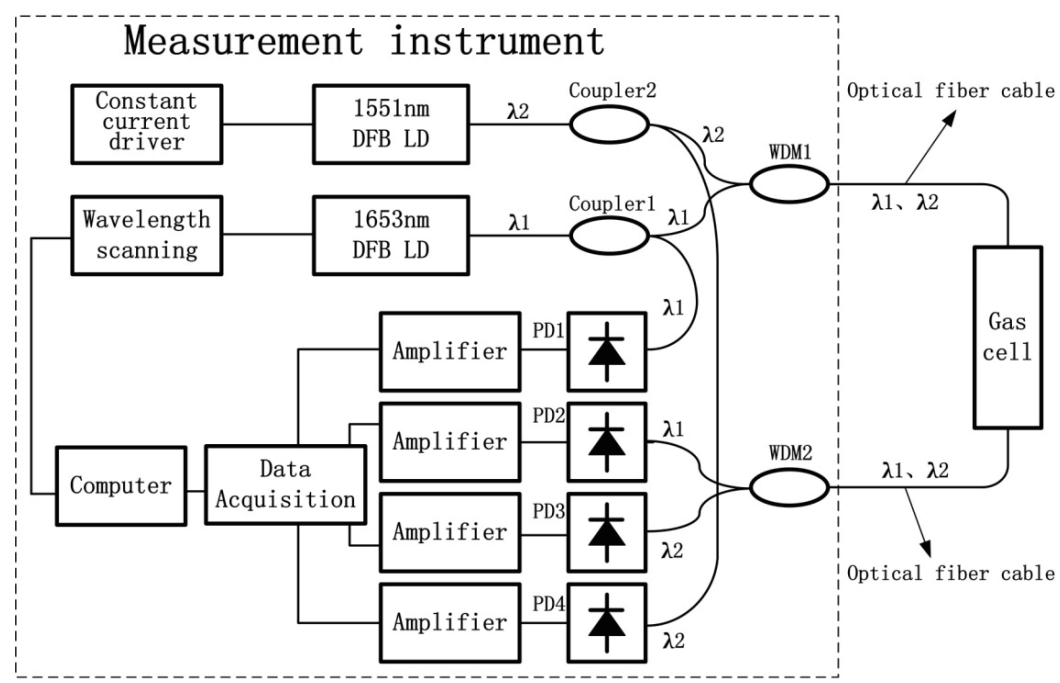

Fig. 1. Schematic diagram of the proposed methane gas sensing system. 
wavelength of $1653 \mathrm{~nm}$ varies with the wavelength scanning when heating or cooling the LD. At the reference wavelength of $1551 \mathrm{~nm}$, the intensity and wavelength of the DFB LD are kept constant. However, a LD will decay during its lifetime; thus, we also monitor the intensity of the reference LD during the measurement.

According to the Beer-Lambert law, when a light beam transmits through a gas cell, the intensity of transmission light is expressed as ${ }^{(4,12)}$

$$
I(\lambda)=I_{0}(\lambda) \exp [-\alpha(\lambda) c l]
$$

where $I(\lambda)$ is the intensity of transmission light, $I_{0}(\lambda)$ is the intensity of incident light, $\alpha(\lambda)$ is the absorption coefficient of the gas at wavelength $\lambda$ in unit concentration and unit length, $l$ is the length of the gas cell in which the light interacts with the gas, and $c$ is the concentration of the gas that we are concerned with.

However, the intensity loss alone in the transmission fiber link can be changed by many other causes aside from the absorption of methane, for example, the loss of connectors, bending loss, and loss in the gas cavity introduced by dust and vapor. Thus, the transmission light is in fact described as

$$
I(\lambda)=I_{0}(\lambda) \exp [-\alpha(\lambda) c L+\beta(\lambda)]
$$

where $\beta(\lambda)$ describes the intensity fluctuation. To monitor the intensity fluctuation of the light source, part of the light emitted from the light source, $I_{d}(\lambda)$, is detected, and the ratio is

$$
R(\lambda)=\frac{I(\lambda)}{I_{d}(\lambda)}=K(\lambda) \exp [-\alpha(\lambda) c L+\beta(\lambda)]
$$

where $k(\lambda)$ is the split ratio, which is a fixed value at a fixed wavelength.

To remove the intensity influence $\beta(\lambda)$, which is induced in the leading fiber and the gas cell, another reference light with a different wavelength is introduced in the measuring system. The wavelength of the reference light is fixed and is not on the absorption band. The reference wavelength is close to the measurement wavelength, so the losses at the two wavelengths caused by bending fiber, dust, and vapor are regarded as the same. Thus, the ratios at the two wavelengths are described as

$$
\begin{gathered}
R\left(\lambda_{1}\right)=\frac{I\left(\lambda_{1}\right)}{I_{\mathrm{d}}\left(\lambda_{1}\right)}=K\left(\lambda_{1}\right) \exp \left[-\alpha\left(\lambda_{1}\right) c L+\beta\left(\lambda_{1}\right)\right], \\
R\left(\lambda_{2}\right)=\frac{I\left(\lambda_{2}\right)}{I_{\mathrm{d}}\left(\lambda_{2}\right)}=K\left(\lambda_{2}\right) \exp \left[-\alpha\left(\lambda_{2}\right) c L+\beta\left(\lambda_{2}\right)\right],
\end{gathered}
$$

where $\lambda_{1}$ is on the absorption line of the $Q$ branch $\left(2 v_{3}\right)$ combination band of methane, and $\lambda_{2}$ is a reference wavelength shifting from $\lambda_{1}$, at which there is no absorption. 
The concentration of methane can be deduced as ${ }^{(13,14)}$

$$
c=\frac{1}{\left[\alpha\left(\lambda_{1}\right)-\alpha\left(\lambda_{2}\right)\right] L}\left\{\ln \frac{K\left(\lambda_{1}\right)}{K\left(\lambda_{2}\right)}-\ln \frac{R\left(\lambda_{1}\right)}{R\left(\lambda_{2}\right)}-\left[\beta\left(\lambda_{2}\right)-\beta\left(\lambda_{1}\right)\right]\right\},
$$

where $\beta(\lambda)$ is the common loss for the two beams caused by dust, transmission loss, connector loss, bending, and so forth. We assume that these losses are the same with the two wavelengths when the two beams go through the same optical path, so we have $\beta\left(\lambda_{1}\right)=\beta\left(\lambda_{2}\right)$. Equation (6) can be simplified as

$$
c=\frac{1}{\left[\alpha\left(\lambda_{1}\right)-\alpha\left(\lambda_{2}\right)\right] L}\left[\ln \frac{K\left(\lambda_{1}\right)}{K\left(\lambda_{2}\right)}-\ln \frac{R\left(\lambda_{1}\right)}{R\left(\lambda_{2}\right)}\right] .
$$

As the tuning wavelength is a few nanometers and the reference wavelength is fixed, $K\left(\lambda_{1}\right) / K\left(\lambda_{2}\right)$ can be regarded as a constant. Detectors 1 and 2 have the same characteristics at wavelengths $\lambda_{1}$ and $\lambda_{2}$, so it is reasonable that $K\left(\lambda_{1}\right) / K\left(\lambda_{2}\right)=1$. Hence, the concentration of methane can be simplified as

$$
c=\frac{1}{\left[\alpha\left(\lambda_{1}\right)-\alpha\left(\lambda_{2}\right)\right] L}\left[\ln \frac{R\left(\lambda_{2}\right)}{R\left(\lambda_{1}\right)}\right]
$$

Here, there is no absorption at $\lambda_{2}$, so $\alpha\left(\lambda_{2}\right)=0$. The concentration of methane can be deduced as

$$
\begin{gathered}
c=\frac{1}{\alpha\left(\lambda_{1}\right) L} \ln \frac{1}{S}, \\
S=\frac{R\left(\lambda_{1}\right)}{R\left(\lambda_{2}\right)}=\frac{I\left(\lambda_{1}\right) I_{d}\left(\lambda_{2}\right)}{I_{d}\left(\lambda_{1}\right) I\left(\lambda_{2}\right)} .
\end{gathered}
$$

Thus, by using Eqs. (9) and (10), we obtain the concentration of methane by detecting four output ports.

\section{Experiment}

We have developed the methane measuring system on the basis of the operation principle, as shown in Fig. 2.

Under atmospheric pressure and room temperature conditions, a bottle of standard methane gas of 3.04\% content with the mixture of methane and air is used as the measured gas, and the methane gas is connected to the gas cell with a plastic pipe line. The light beam transmits to the gas cell with a Corning SMF-28 single-mode fiber of $900 \mu \mathrm{m}$ loose tube and $1.0 \mathrm{~m}$ length, and the operation distance of the gas cell is $100 \mathrm{~mm}$ with a loss of $0.55 \mathrm{~dB}$. The $1551 \mathrm{~nm} \mathrm{LD}$ 

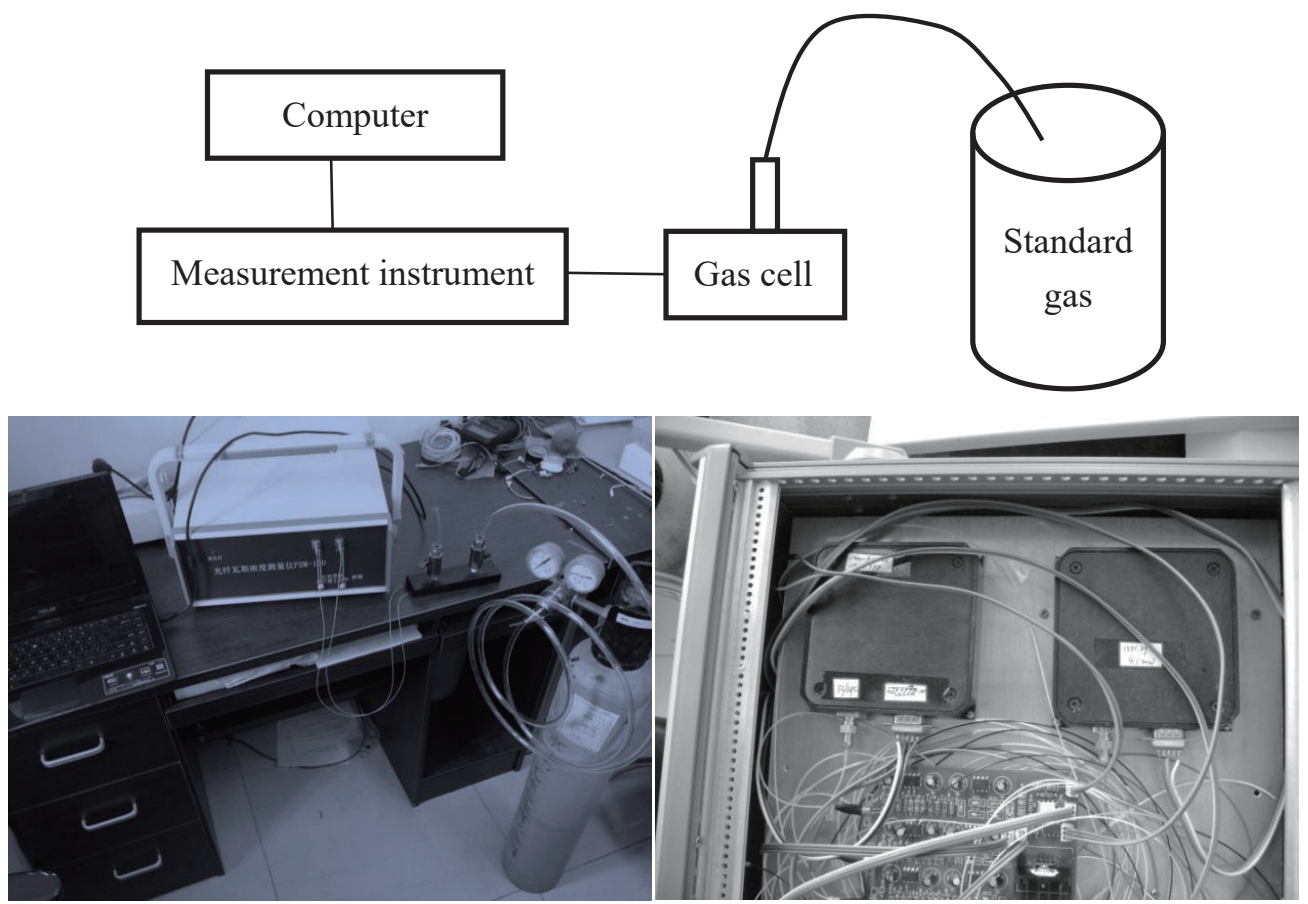

Fig. 2. (Color online) Methane measuring system.

has a power of $6.2 \mathrm{dBm}$ and a line band of $0.01 \mathrm{~nm}$. The $1653 \mathrm{~nm} \mathrm{LD}$ has a power of $4.2 \mathrm{dBm}$ and a line band of $0.01 \mathrm{~nm}$. The spectra of the two LDs are shown in Fig. 3. The operation temperature of the $1551 \mathrm{~nm} \mathrm{LD}$ is fixed at $25^{\circ} \mathrm{C}$. The operation temperature of the $1653 \mathrm{~nm} \mathrm{LD}$ is scanned from 15 to $40{ }^{\circ} \mathrm{C}$, inducing a wavelength shift of $2.5 \mathrm{~nm}$.

We experimentally measured the methane gas of $3.04 \%$ content. Figure 4 shows the power change at the measuring-wavelength channel during one measurement period. Figure 4(a) is the measuring signal, which goes through the gas cell, $I(1653 \mathrm{~nm})$. Figure $4(\mathrm{~b})$ is the power change of the $1653 \mathrm{~nm} \mathrm{LD}, I_{d}(1653 \mathrm{~nm}) . I(1653 \mathrm{~nm})$ is divided by $I_{d}(1653 \mathrm{~nm})$ to remove the power fluctuation caused by temperature scanning, and the result is shown in Fig. 4(c). The background of the result is almost unchanged with temperature scanning. To remove the common loss in the leading fiber and the gas cell, another light of $1551 \mathrm{~nm}$ wavelength is used as the reference beam. During the period of temperature scanning at the $1653 \mathrm{~nm}$ channel, the power change of the $1551 \mathrm{~nm}$ channel is as shown in Fig. 5. This light is also divided into two beams. One beam is detected directly by PD4 to monitor the power change of the $1551 \mathrm{~nm}$ light source, $I_{d}(1551 \mathrm{~nm})$. The other beam, $I(1551 \mathrm{~nm})$, transmits to the gas cell, and is detected by PD3. $I_{d}(1551 \mathrm{~nm})$ is divided by $I(1551 \mathrm{~nm})$, and the result is shown in Fig. 5(c). Finally, 


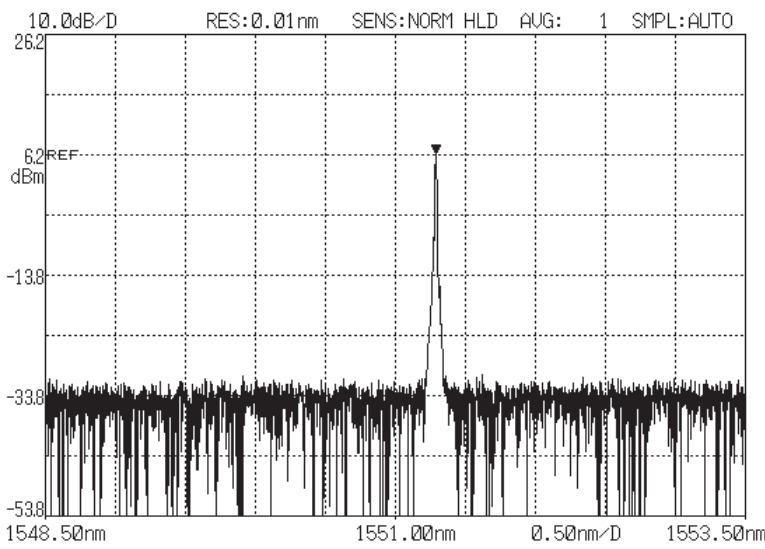

(a)

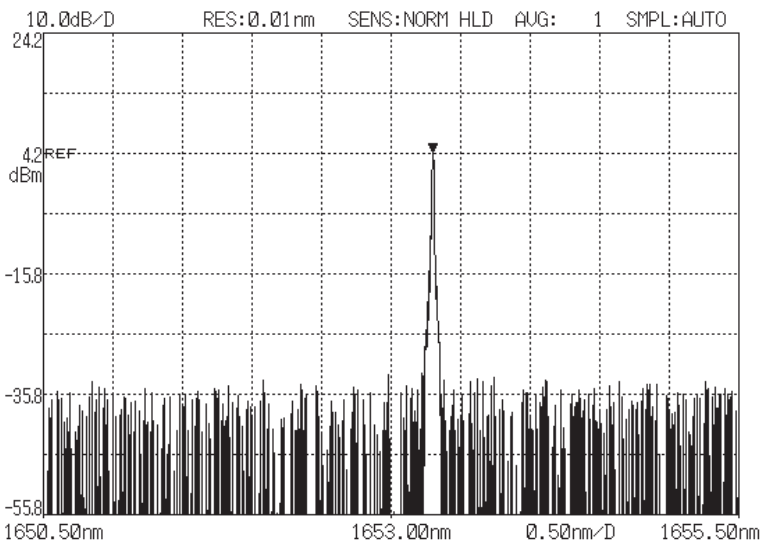

(b)

Fig. 3. Spectra of (a) $1551 \mathrm{~nm} \mathrm{LD}$ and (b) $1653 \mathrm{~nm} \mathrm{LD}$.

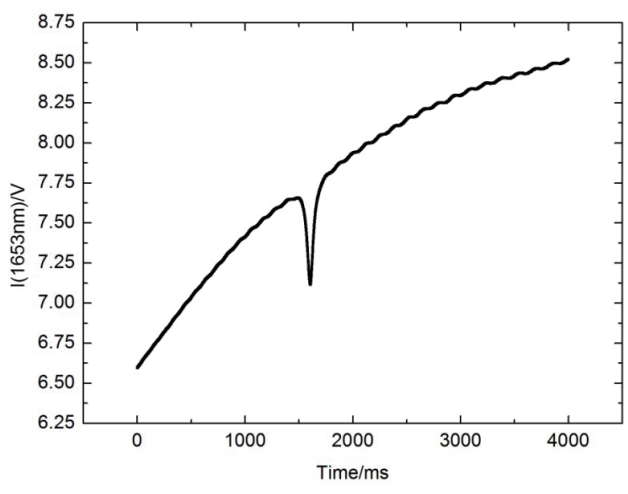

(a)

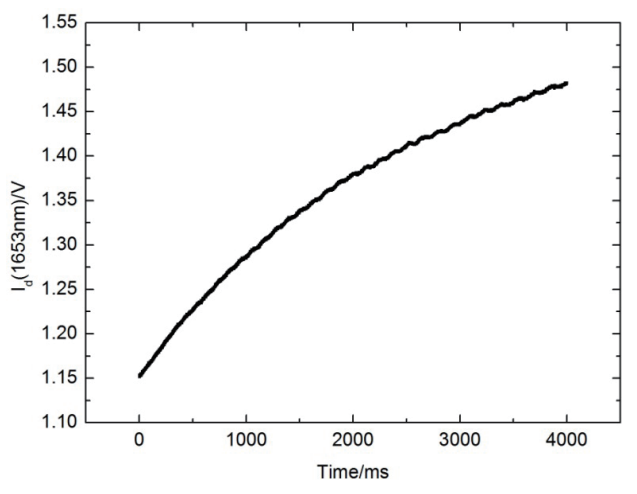

(b)

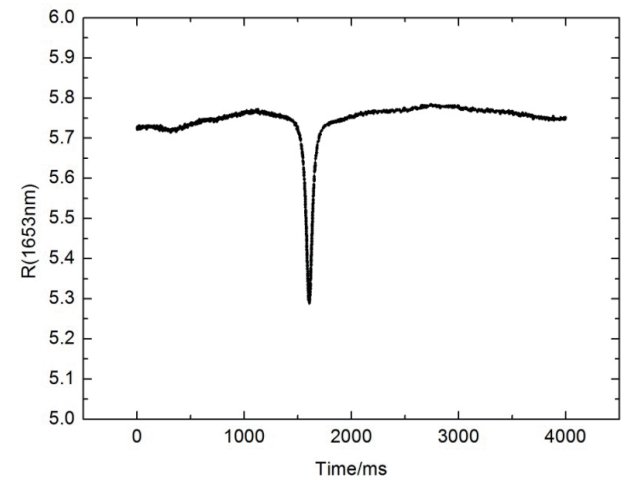

(c)

Fig. 4. Power changes at the $1653 \mathrm{~nm}$ channel. (a) Measuring signal, (b) power change of light source, and (c) removal of power fluctuation caused by temperature scanning.

we obtained the value of $S$ in Eq. (10), as shown in Fig. 6. The depth of the notch in Fig. 6 reflects the content of the methane gas, and the depth is not affected by the loss change of the transmission link fiber, dust, vapor, and so forth.

In another experiment, we investigated the value of $R\left(\lambda_{1}\right)=I\left(\lambda_{1}\right) / I_{d}\left(\lambda_{1}\right)$, which changed with increasing scanning temperature. The LD chip is mounted on a thermal electric cooler (TEC), 


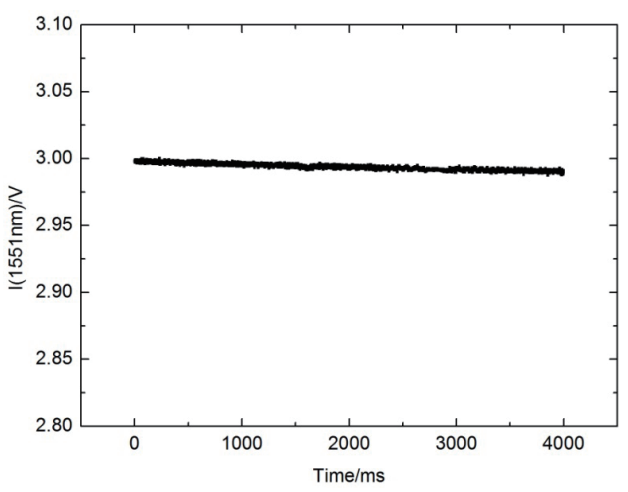

(a)

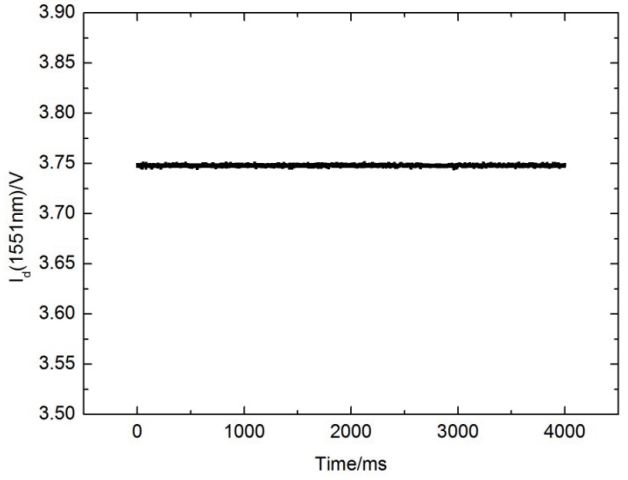

(b)

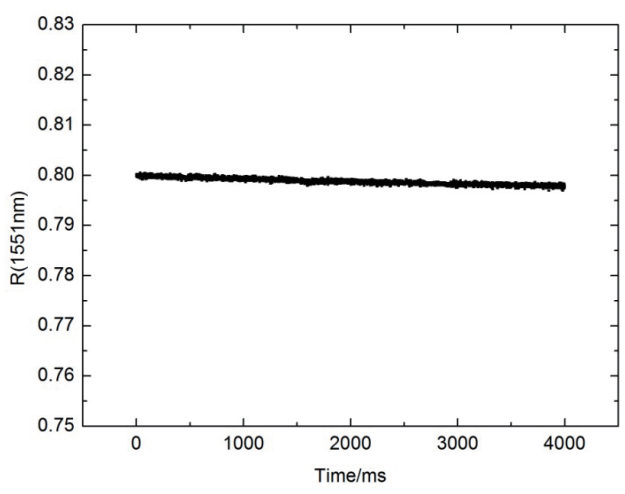

(c)

Fig. 5. Power changes at the $1551 \mathrm{~nm}$ channel. (a) Measuring signal, (b) power change of light source, and (c) power fluctuation during period of temperature scanning.

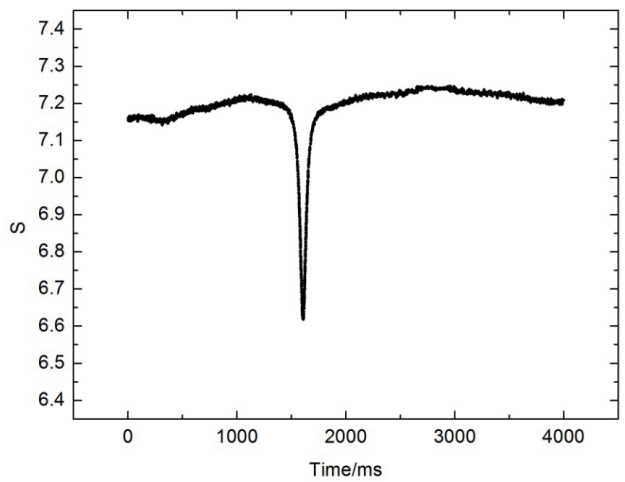

Fig. 6. Value of $S$ in Eq. (10).

and a thermistor with a value of $10 \mathrm{k} \Omega$ is used to measure the temperature. When cooling or heating the LD chip using the TEC, the operation temperature of the LD chip is changed. Figure 7 shows the result of $R\left(\lambda_{1}\right)$ when heating or cooling. As temperature increases, there is an absorption valley at $36{ }^{\circ} \mathrm{C}$. As temperature decreases, the absorption valley occurs again, and the depth is the same as that when temperature increases. Heating or cooling the LD 


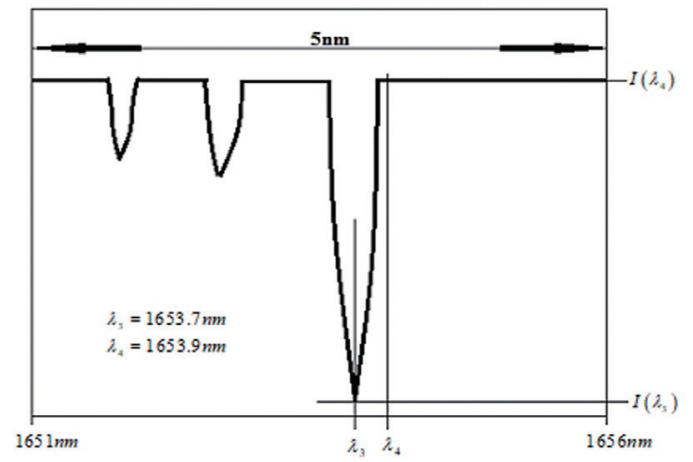

(a)

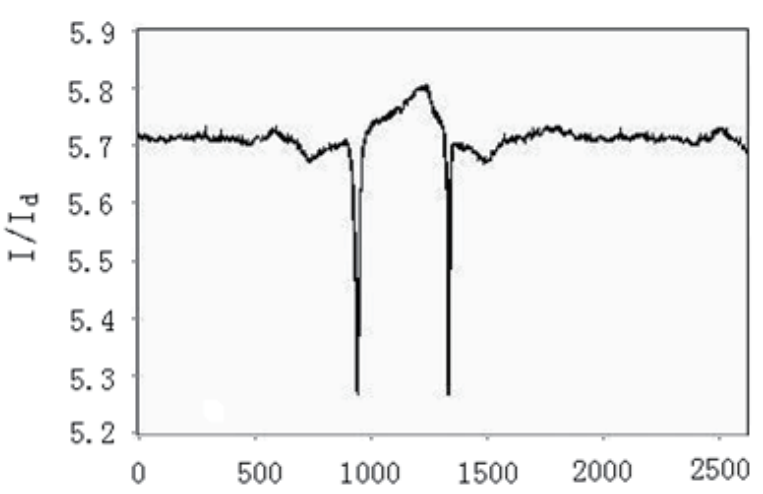

(b)

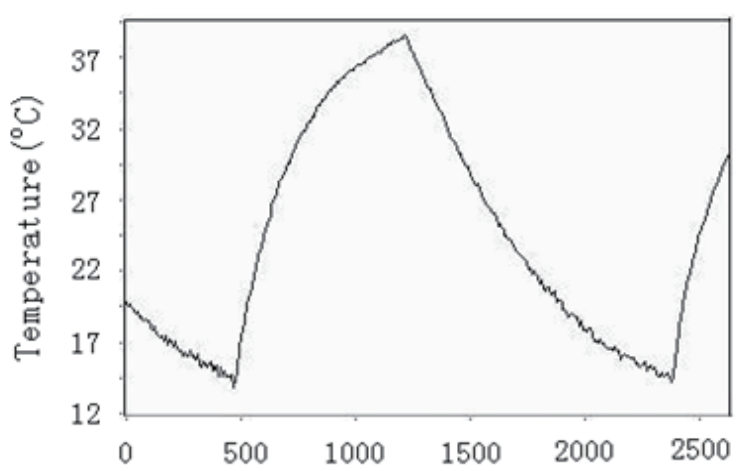

(c)

Fig. 7. $\quad R\left(\lambda_{1}\right)$ changed with the scanning temperature, (a) schematic output of methane measurement employing wavelength scanning based on temperature control techniques, (b) $R\left(\lambda_{1}\right)$, and (c) temperature change.

chip in fact changes the output wavelength of the LD. At $36{ }^{\circ} \mathrm{C}$, the output wavelength of the $\mathrm{LD}$ is aimed at an absorption line of the methane gas, resulting in an absorption valley at this temperature.

We continually measured the methane gas of $3.04 \%$ content, and the values of the absorption valley are shown in Fig. 8. We can determine that $\Delta S \leq 0.016677$, and the standard error $\sigma$ is $\approx 3.65286 \times 10^{-3}$. Thus, the measurement resolution $2 \sigma$ of $7.30571 \times 10^{-3}$ can be obtained for measuring methane of $3.04 \%$ content. Thus, we can determine that the measurement resolution for methane gas is $0.038 \%$. Because we have only one standard gas in our laboratory, we have no ability to measure the change in the absorption valley with different methane contents. However, a high measurement resolution of $0.038 \%$ can be reached by using continuous measurements.

We measured the methane content in liquid natural gas (LNG), and the results of $R(1551$ $\mathrm{nm}), R(1653 \mathrm{~nm})$, and $S$ are shown in Fig. 9. It is clear that methane gas concentration in LNG is low. By comparing the values of $S$ for the LNG and standard methane gas of $3.04 \%$ content, we determined that the methane content in LNG is $0.63 \%$. This is consistent with the actual value, because the main component of LNG is butane. 


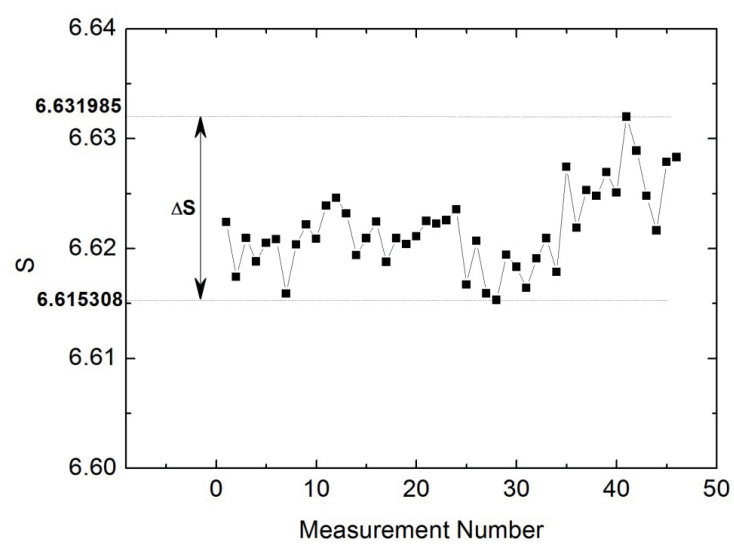

Fig. 8. Results of continuous measurements.

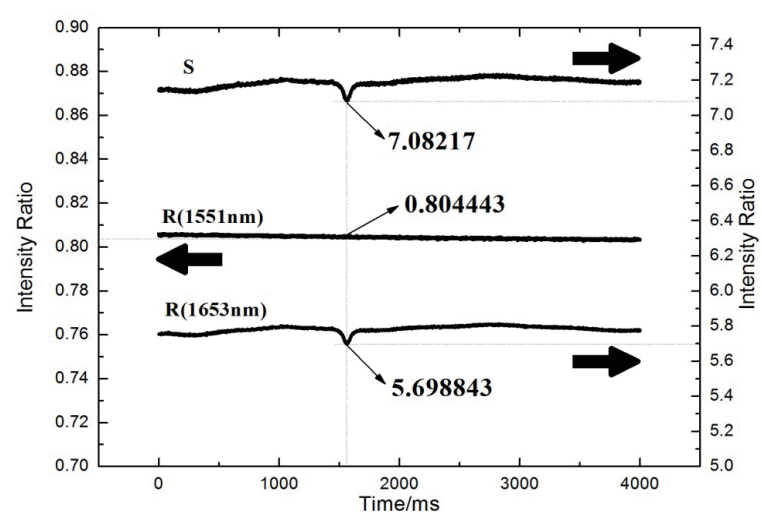

Fig. 9. Measurement results of LNG.

\section{Conclusion}

In conclusion, we have demonstrated a fiber optic methane sensing system based on the temperature-tunable DFB laser. Two LDs with different wavelengths are used in the system. One LD operates in the wavelength scanning mode, and the scanning wavelength covers the absorption line of methane gas. The wavelength scanning is realized by tuning the operation temperature of the LD. The other LD is used as the reference beam to eliminate the fluctuation of light intensity, the wavelength of which is not fixed on the absorption line. By comparing the intensities of the two wavelengths during wavelength scanning, we can obtain the content of the methane gas. Experimental results show that a measurement resolution of $0.038 \%$ can be reached. The above measuring system has the advantages of high precision, low cost, and high reliability.

\section{Acknowledgments}

This work is mainly supported by the scientific and technological support projects of Sichuan Provincial Government (No. 2011GZ0254), the key research projects of Science and Technology Plan for Yibin Municipal Government (No. 2013GY021), the construction plan projects for scientific research and innovation team of Sichuan Provincial Department of Education (No. 15TD0031), and the doctoral fund projects of Chengdu Technological University (No. 2016RC005).

\section{References}

1 H. Inaba, T. Kobayasi, and M. Hirama: Electron. Lett. 15 (1979) 749.

K. Chan, H. Ito, and H. Inaba: Appl. Opt. 23 (1984) 3415.

3 Z. Wang, J. N. Cao, and K. K. Zhang: Acta Photon. Sinica 40 (2011) 255.

4 S. H. Xiao and J. Tang: Opt. Tech. 38 (2012) 555.

5 S. H. Xiao, Y. Jiang, and D. Liu: Microwave Opt. Technol. Lett. 55 (2013) 2117. 
6 Y. F. Shen, Y. J. Ma, and H. Fu: Chin. J. Sens. Actuators 27 (2014) 704.

7 D. Jacob, N. H. Tran, F. Bretenaker, and A. L. Floch: Appl. Opt. 33 (1994) 3261.

8 Y. Shimose, T. Okamoto, A. Maruyama, and M. Aizawa: IEEE Photon. Technol. Lett. 3 (1991) 86.

9 Y. Jiang and C. J. Tang: Sens. Actuators, B 133 (2008) 174.

10 B. Zhou and Z. Guan: Proc. Asia Optical Fiber Communication \& Optoelectronics Conf. (IEEE, 2007) 296298.

11 K. Yu, C. Wu, and Z. Wang: IEEE Sens. J. 10 (2010) 728.

12 H. Tai, K. Yamamoto, M. Uchida, and S. Osawa: IEEE Photon. Technol. Lett. 4 (1992) 804.

13 S. H. Xiao, C. J. Tang, and Y. Jiang: Acta Photon. Sinica 41 (2012) 1479.

14 S. H. Xiao, Y. Q. Jing, and J. Tang: Chin. J. Sens. Actuators 30 (2017) 162. 